\title{
CAVITATION EROSION WEAR OF METALLIC SPECIMENS USING THE NEW COMPACT ROTATING DISK DEVICE
}

\author{
G. Bazanini, | ABSTRACT \\ J. D. Bressan, \\ and M. A. Klemz \\ Santa Catarina State University-Udesc Joinville \\ Department of Mechanical Engineering \\ R.Tenente Antonio Joao, s/n \\ Campus Universitário \\ Joinville-SC, Brazil \\ 89223-100 \\ dem2gb@joinville.udesc.br \\ A more compact version of the rotating disk device (where a disk with \\ cavitation inducers and specimens fixed on it rotates in water to provide \\ cavitating flow) is used here to study the failure by cavitation in metallic \\ specimens. The damage in the specimens is measured by mass loss versus \\ exposition time to cavitating flow diagrams. Images of the test specimens \\ obtained by electronic microscope are also shown and discussed. After 25 \\ hours working in cavitating conditions, aluminum, brass and cast iron are \\ weared by the cavitation phenomenon, resulting in pitting formation and \\ mass loss. After each 5 hours operating in cavitating conditions, the \\ specimens are cleaned by ultrasound, dried and weighted in a digital balance \\ to obtain the mass loss in the process by comparing to its initial weight. All \\ specimens, aluminum, brass and cast iron, were eroded by cavitation \\ although in the aluminum the mass loss was lesser than the expected. The \\ brass specimen was eroded as expected, mas no mass loss could be attained \\ for the cast iron due to specimen oxidation. \\ Keywords: cavitation, pits, erosion
}

\section{NOMENCLATURE \\ $P$ pressure \\ $R$ bubble radius \\ $S$ surface tension}

\section{Subscripts \\ $L \quad$ liquid \\ $v$ vapor}

\section{INTRODUCTION}

The cavitation phenomenon, that is, the formation, growth and collapse of air and vapor bubbles in liquids is, as well known, responsible for damage in metallic and non-metallic solid structures in liquid mediums, remarkably in water. Such bubbles, or cavities, nucleate from micro-bubbles of

air present in the liquid medium. This bubble formation phenomenon is named

"cavitation inception" (Hammitt, 1980). The growth process involves primarily the action of pressure forces, but these are the result of the interplay of surface action, inertia and viscosity, and also gas diffusion and evaporation (Bazanini and Hoays, 2008). In most simple terms, the conclusion results from the static force balance at the bubble wall between the surface tension and pressure differential:

$$
P_{V}-P_{L}=\frac{2 S}{R}
$$

where $P$ signifies pressure at the bubble wall, the subscript $v$ signifies vapor (and/or gás) and the subscript $L$ signifies liquid. $S$ is the surface tension, and $R$ is the bubble radius.

As it growths, the bubble is filled by the liquid vapor, present when the pressure and temperature of the mixture are close to the vapor pressure and temperature of the surrounding liquid, until reaching an equilibrium radius as can be seen in Young (1989). After that, the bubble or bubbles, begin their collapse and may disappear or initiate a new cycle, that may then be repeated for several times until the bubbles disappear (Young, 1989).

When bubbles collapse near solid surfaces, damage may occur. The most usual example is the cavitation erosion in centrifugal pumps (Hattori; Kishimoto, 2008). Two are the already known damage mechanisms: liquid micro-jets impingement and shock waves (Fujikawa; Akamatsu, 1980). The micro-jets are caused by bubble splitting in two parts near the solid surface (influenced by that surface close to it; a good visualization of the phenomenon can be seen in Shervani-Tabar et al. (2003) in the final stages of collapse; shock waves are caused by instant (collapse duration is of about miliseconds) variable pressure fields (Bazanini, 2003). These micro-jets and shock waves are responsible for loss material from the surface, basically by fatigue failure.

With the goal to study the effects of cavitation on solid surfaces, several devices have been developed along the last decades, such as the cavitation test chamber (Steller et al., 2005). Some of them are the jet-impact damage device (Janikaram, 1973) that consists of water liquid jets 
impinging in specimens fixed on rotating disks, the vibratory apparatus (Knapp et al., 1970) where the specimens are set to vibrate in the test liquid, the vertical (related to the axis) rotating disk (Wood et al., 1967), where a disk with the specimens fixed on it is rotating in water to provide cavitating flow, and finally (the most important, because it reproduces the phenomenon of the flow through a pump) is the horizontal rotating disk device (Rao et al., 1980; Vivekananda, 1983; Zhiye, 1983; Zhang et al., 1996).

\section{EXPERIMENTAL SET UP AND APPARATUS}

The device consists of a water chamber in which a metallic disk rotates. On the disk surface are located the cavity inducers, that may be holes or protruding pins, and the specimens as well. The disk is fixed on the shaft and may be detached to switch the specimens. A glass cover is mounted on the chamber to visualize the flow and the bubble formation inside.

The purpose of the device is to create the bubbles that will be responsible for the erosion of the specimens fixed on the disk surface and close to the inducers. To prevent vibration problems, the holes and the specimens are situated on opposite radial positions of their reciprocals. Vibration absorbers are also used at the equipment foundations, and the disk with the specimens is balanced before tests were performed. Details of the rotating disk with the specimen positions can be seen in Fig. 1 below.

The peripheral velocity is function of the specimen position on the disk, since the rotation was kept constant at $4400 \mathrm{rpm}$. The peripheral velocity associated to each position is listed below on Table 1.

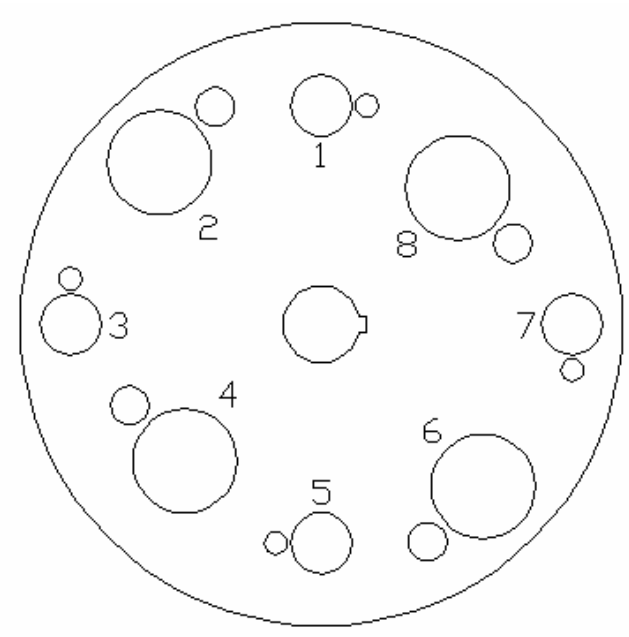

Table 1. Peripheral velocities.

\begin{tabular}{|c|c|}
\hline Disk position & $\begin{array}{c}\text { Peripheral } \\
\text { velocity }(\mathrm{m} / \mathrm{s})\end{array}$ \\
\hline 1 & 41,8 \\
\hline 2 & 43,7 \\
\hline 3 & 47,9 \\
\hline 4 & 36,8 \\
\hline 5 & 41,8 \\
\hline 6 & 43,7 \\
\hline 7 & 47,9 \\
\hline 8 & 36,8 \\
\hline
\end{tabular}

Some authors (Rao et al., 1980; Vivekananda, 1983) used a cast iron chamber and protruding pins mounted on the disk. We are now using holes in the disk as inducers, and the disk and the chamber are made by stainless steel, more resistant to cavitation erosion. It is being made a compact version of the device, that is, smaller chamber and disk diameter (250 $\mathrm{mm})$, as well as a shorter shaft. By using the "Intermediary Device", commonly used to connect pumps to electric motors, we can avoid the use of the bearing and the coupling, resulting in a shorter shaft. That is, the chamber now replaces the pump. This reduces loss transmissions as well as alignment problems. The Intermediary Device is made by cast bronze to prevent corrosion. Figure 2 shows that device and the chamber itself. Connections for inlet and outlet of water, temperature and pressure visualizations, and water drain and air outlet are also provided. Eight baffles equally spaced are welded in the chamber (and at a distance $18 \mathrm{~mm}$ from the disk) to kept bubbles to collapse over the test specimens. A frequency inverter is used to control the motor operation, and thus the disk rotation.

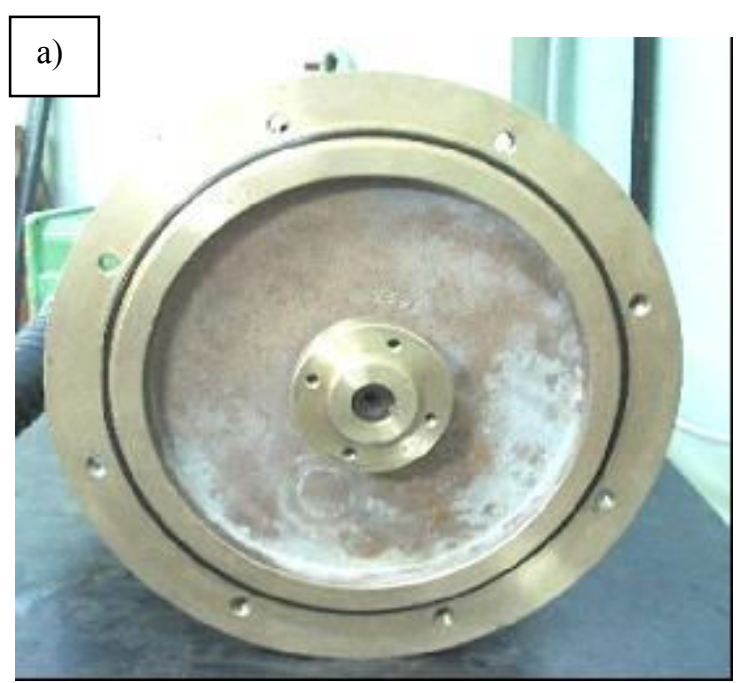

Figure 1. Rotating disk with specimen positions 


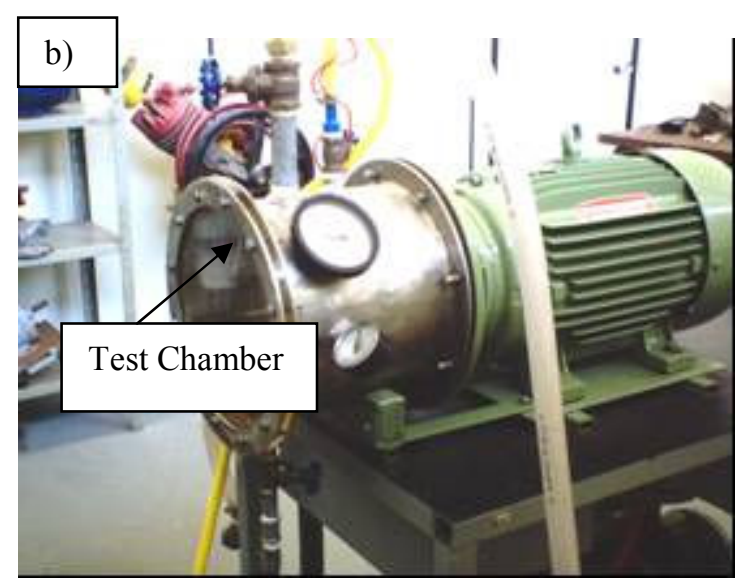

Figure 2. a) intermediary device. b) test device.

Several test specimens are showed in Fig. 3a below. In these tests, cast iron, aluminum and brass were used. To fix the disk to the to electric motor shaft, a flange was specially manufactured in bronze. That can be seen in Figure $3 \mathrm{~b}$.
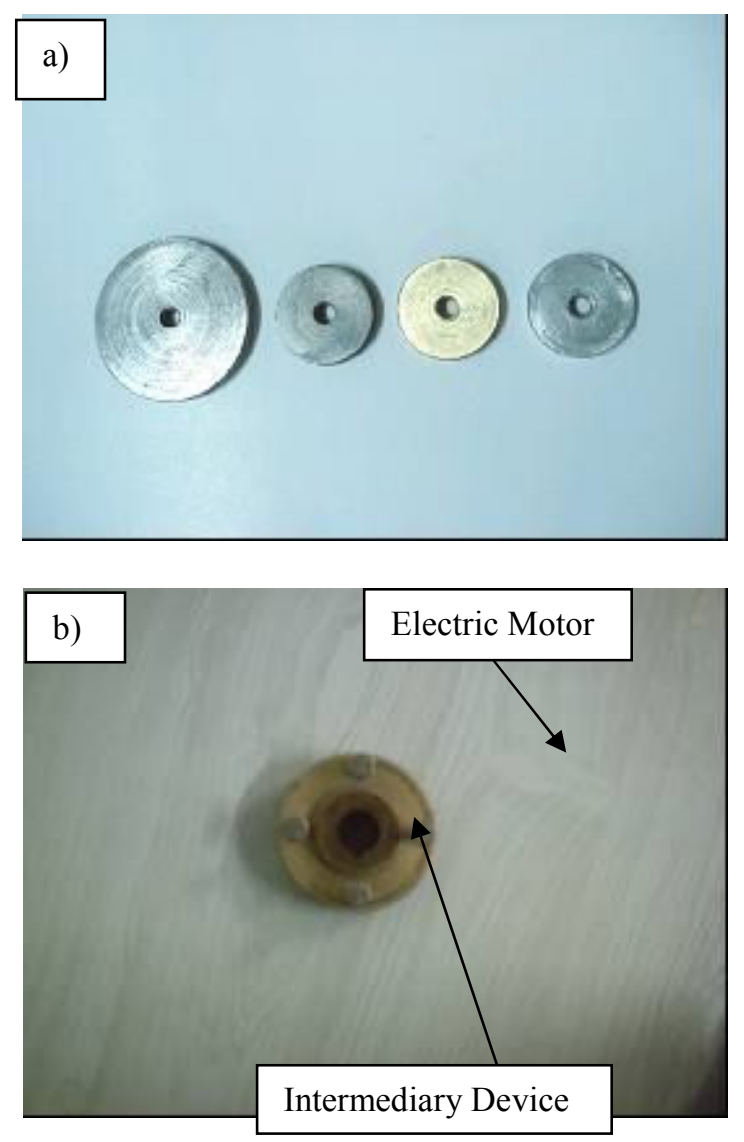

Figure 3. a) test specimens. b) flange used to fix the disk.

For cooling purposes, a water reservoir is used to circulate the water in the chamber, being the piping provided by control valves and a filter to protect the pump from small particles resulting from the erosion.

\section{EXPERIMENTAL PROCEDURE}

Inlet and outlet valves are used to regulate temperature and pressure in the chamber that is connected to an overhead water reservoir. Temperature and pressure in the chamber are monitored by a thermometer and a manometer of scales $0-100{ }^{\circ} \mathrm{C}$ and $0-3$ bar, respectively. The speed of the electric motor (and then the water flow velocity on the specimens) is controlled by the frequency inverter, that may also be used to see the power consumption.

After each 5 hours operating in cavitating conditions, the specimens are cleaned by ultrasound, dried and weighted in a digital balance to obtain the mass loss in the process by comparing to its initial weight. Images of the specimens were also obtained using an electronic microscope.

\section{RESULTS AND DISCUSSIONS}

This version of the rotating disk device was very effective in bubble formation as can be seen in Fig. 4 , where it is possible to see bubbles formation inside the chamber. The volume occupied by the bubbles in the chamber was approximately $5 \%$ of the chamber volume, measured after the equipment is shut off. The cooling water consumption was of 8 liters per minute.

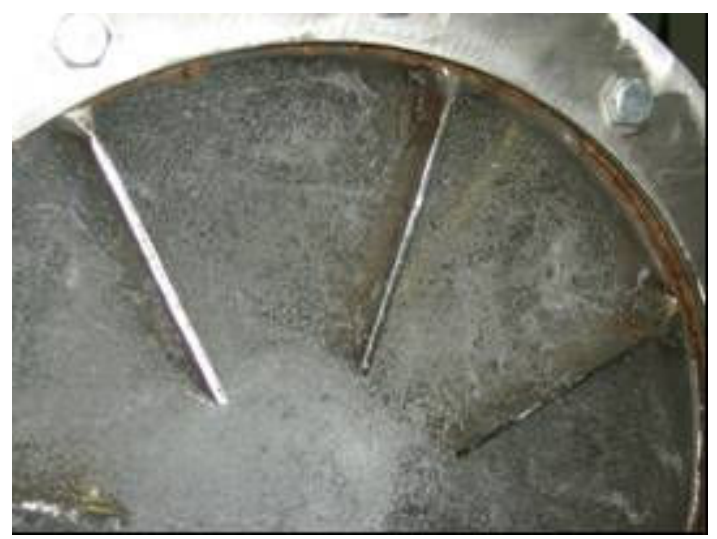

Figure 4. Bubbles formation

In these experiments, commercial aluminum and common brass of 2.54 centimeters in diameter and cast iron of 4.34 centimeters in diameter were tested as specimens fixed on the disk. Table 2 shows the characteristics of this device in comparison with other existing ones. The equipment was first tested at $3600 \mathrm{rpm}$. After that, it was operated at $4400 \mathrm{rpm}$ (resulting in a flow velocity at the specimen of 47.9 $\mathrm{m} / \mathrm{s}$ for the aluminum and the brass, and of $36.8 \mathrm{~m} / \mathrm{s}$ for the cast iron) during 25 hours to obtain the results presented here. This experiment was realized 
at atmospheric pressure, and the temperature was kept at $37 \cdot \mathrm{C}$ by water flow circulation.

After 25 hours of operation, it was observed a mass loss of $36.2 \mathrm{mg}$ for the brass. The mass loss of the brass is close to those obtained by Vivekanada (1983) and Rao et al. (1980) who used the same device, working with a rotating disk of $335 \mathrm{~mm}$, and using pins as cavitation inducers. Zhiye (1983), otherwise, used holes as cavitation inducers in a disk of $350 \mathrm{~mm}$ of diameter. The mass losses as well as the operating conditions can be seen in Table 3 .

Table 2. Comparison among existing devices.

\begin{tabular}{|c|c|c|c|c|}
\hline Work & $\begin{array}{c}\text { Electric } \\
\text { motor } \\
\text { speed } \\
(\mathrm{rpm})\end{array}$ & $\begin{array}{c}\text { Electric } \\
\text { motor } \\
\text { power } \\
(\mathrm{kw})\end{array}$ & $\begin{array}{c}\text { Disk } \\
\text { diameter } \\
(\mathrm{mm})\end{array}$ & $\begin{array}{c}\text { Cavitation } \\
\text { inducer }\end{array}$ \\
\hline Present work & 4400 & 22 & 250 & holes \\
\hline $\begin{array}{c}\text { Rao et al., } \\
1980\end{array}$ & 2925 & 26 & 335 & pins \\
\hline $\begin{array}{c}\text { Vivekananda } \\
\text { et al., 1983 }\end{array}$ & 2880 & 26 & 335 & pins \\
\hline Zhiye, 1983 & 2950 & 30 & 350 & holes \\
\hline
\end{tabular}

For an accurate comparison, all tests should be made in identical conditions, that is, the same inducers, peripheral velocity, and the same specimens (sizes

Table 3. Mass loss for brass and materials). Although Vivekananda (1983) worked with greater specimens diameters $(6.35 \mathrm{~cm})$, he used smaller peripheral velocities $(38 \mathrm{~m} / \mathrm{s})$. Larger surface areas

\begin{tabular}{|c|c|c|c|c|}
\hline Work & $\begin{array}{c}\text { Mass loss } \\
(\mathrm{mg}) / \text { hours } \\
\text { in } \\
\text { cavitating } \\
\text { condition }\end{array}$ & $\begin{array}{c}\text { Peripheral } \\
\text { velocity } \\
(\mathrm{m} / \mathrm{s})\end{array}$ & $\begin{array}{c}\text { Specimen } \\
\text { diameter } \\
(\mathrm{mm})\end{array}$ & $\begin{array}{c}\text { Cham- } \\
\text { ber } \\
\text { tempe- } \\
\text { rature } \\
(\cdot \mathrm{C})\end{array}$ \\
\hline $\begin{array}{c}\text { Present } \\
\text { work: }\end{array}$ & $\begin{array}{c}6.2 / 5 \\
36.2 / 25\end{array}$ & 47.9 & 25.4 & 37 \\
\hline $\begin{array}{c}\text { Rao et al., } \\
1980\end{array}$ & $42.5 / 25$ & 53.8 & 63.5 & 34 \\
\hline $\begin{array}{c}\text { Zhiye, 1983 } \\
\text { Zivekanand, } \\
1983\end{array}$ & $30 / 20$ & 38.0 & 63.5 & 32 \\
\hline $\begin{array}{c}\text { Vivet } \\
\text { informed }\end{array}$ & 26 \\
\hline
\end{tabular}

lead to a greater mass loss while smaller peripheral velocities have the opposite effect. Rao et al. (1980) worked with greater specimens diameters and greater peripheral velocities obtaining, as expected, a greater mass loss for the same period of time. Zhiye (1983) conducted his experiments for 250 minutes only (approximate 4.2 hours), and the results are very close to the present work, although the specimen diameters are not informed in that work. Figure 5 below shows the power consumption for the disk without the cavitation inducers.

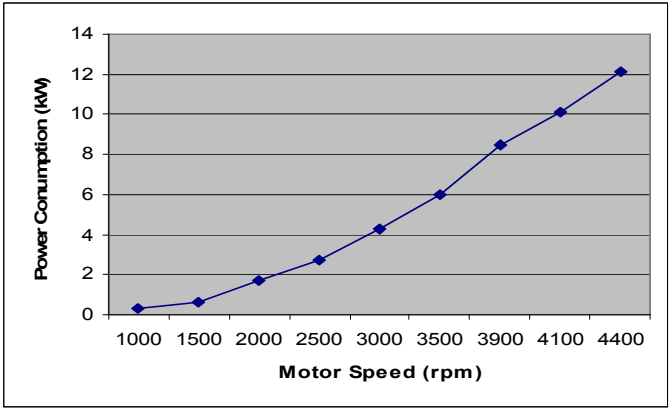

Figure 5. Disk power consumption.

The specimens were also analyzed by scanning electronic microscope. The images obtained are in Figs. 6 to 8 below. Figure 6 was obtained before the erosion tests, for the aluminum specimen.

Cavitation pits can be identified in Figure 7a for the aluminum specimen. Erosion by cavitation was also observed in the brass specimens. Such erosion regions can be clearly seen in Figures $7 \mathrm{~b}$ and 8 .

The erosion rate of specimens with time under cavitating conditions is shown as mass loss in function of time diagrams in Figures 9 and 10, for the materials tested.

For the cast iron (Figure 9), while by one side there is a loss of the specimen mass by cavitation, on the other hand there is a mass gain by oxidation of the specimen surface. That explains the increase of the mass registered with time for both specimens tested.

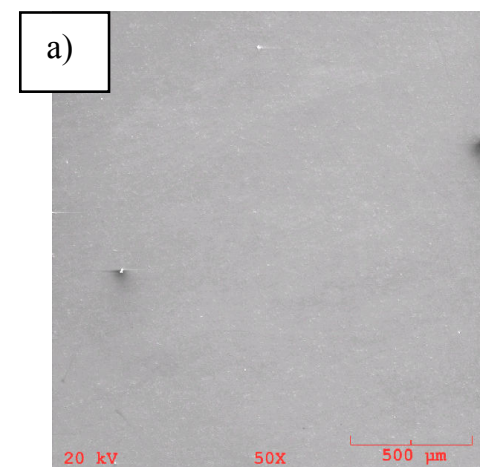

\section{b)}

Figure 6. a) aluminum specimen before testing: magnification 50x. b) magnification 500x 

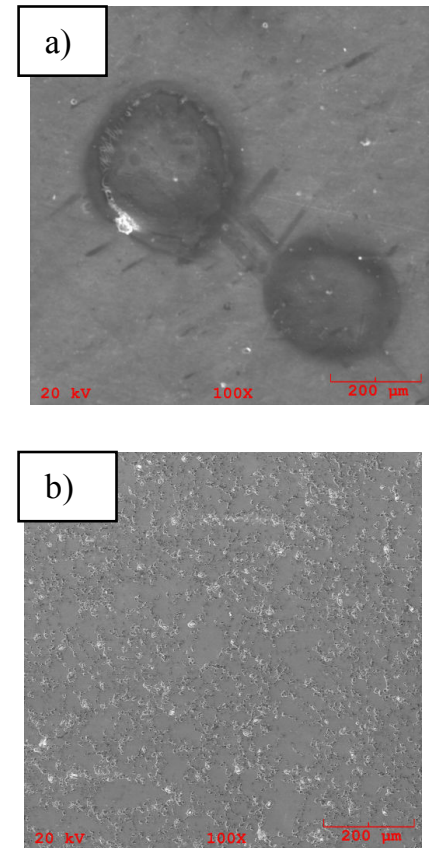

Figure 7. a) aluminum, cavitation pit, magnification 100x, after 10 hours. b) cavitation erosion, brass, magnification 100x, after 5 hours.
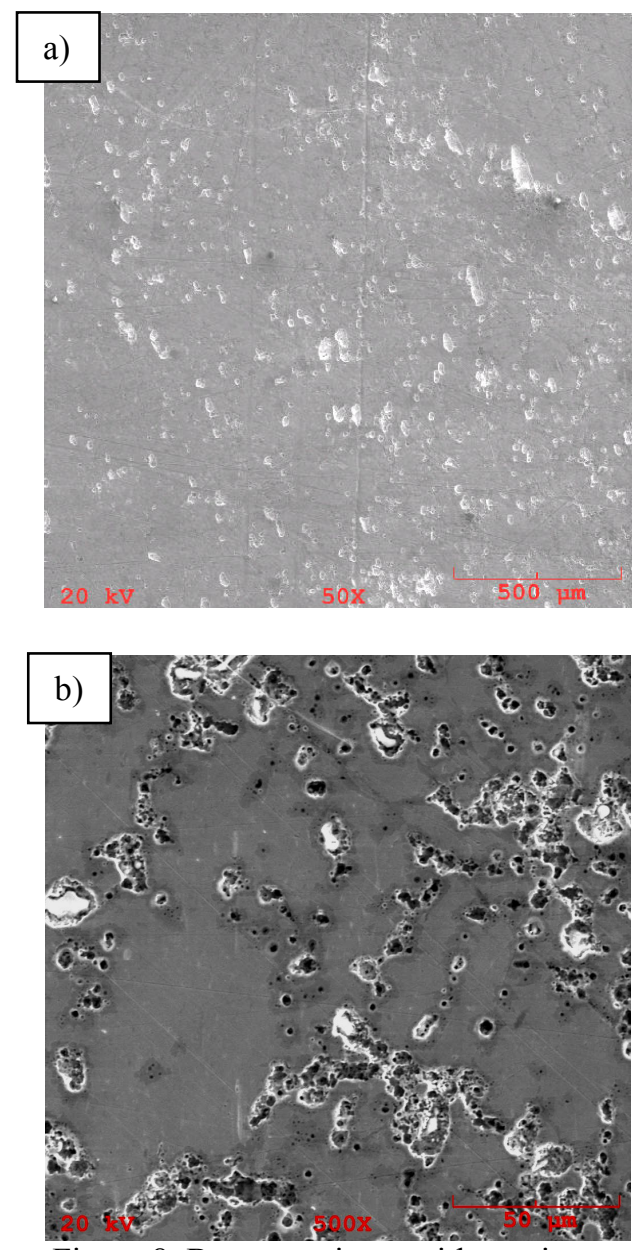

Figure 8 . Brass specimen with erosion cavitation: a) magnification 50x. b) magnification 500x.

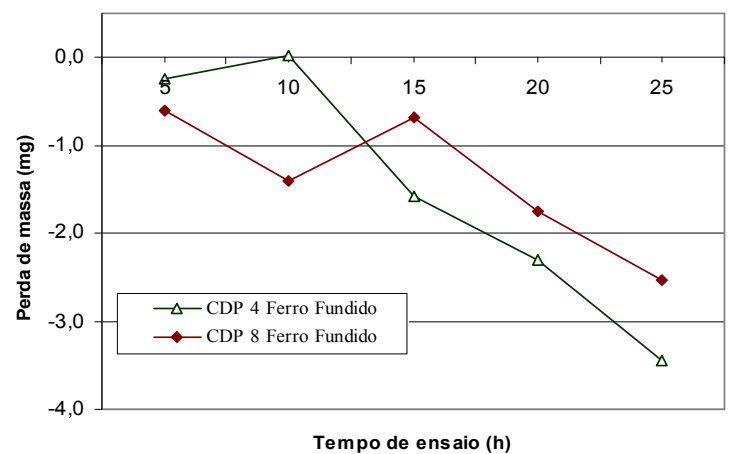

Figure 9. Mass loss curves for the cast iron specimens.

For the brass, the mass loss by erosion was the expected one. It was very close to the existing results in the references, as can be seen in table 3 . It was expected a greater mass loss for aluminum in relation to brass, as can be seen in previous works about cavitation erosion as Soyama (2001). However, there was a roughening of the surface by plastic deformation with a very low mass loss in the aluminum specimens. Cavitation damage without mass loss is also expected and regarded as erosion (ASM Handbook, 1992).

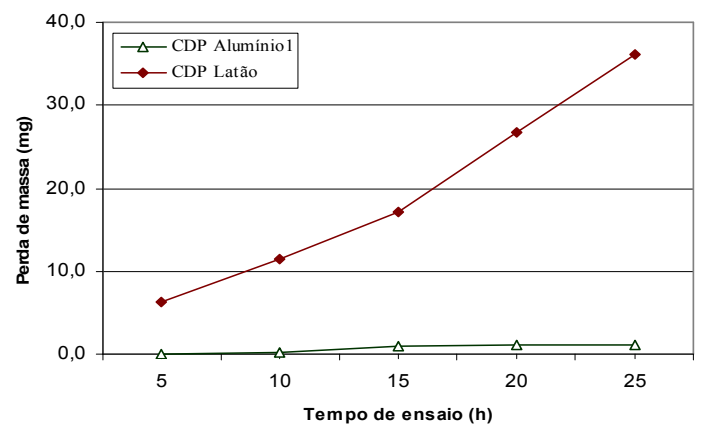

Figure 10. Mass loss curves for aluminum and brass specimens.

\section{CONCLUSIONS}

The apparatus used here is more compact then the existing ones. It has a shorter shaft, avoiding the use of the bearing and the coupling. That results in a gain in the power transmitted, since power transmission losses are reduced. It also has smaller dimensions then the preceding ones: smaller disk diameter resulting in a smaller chamber volume. That also results in a reduction of the power consumption. Such small dimensions allow us to connect the chamber right to the electric motor only through the use of the intermediary device. The equipment worked quite well by producing bubbles and the correlated erosion damages in the specimens surfaces, and no failure in the equipment happened during its operation at $4400 \mathrm{rpm}$. 
Comparing Table 3 and Figure 5, we conclude that the power consumed in the inducers at $4400 \mathrm{rpm}$ to generate the bubbles was about $10 \mathrm{~kW}$.

The pittings and erosion resulting from cavitation could be observed with the aid of an electronic microscope and can be seen in Figs. 7 and 8 .

For the cast iron, it was not possible to detect the mass loss since, while there is an increase in the specimen mass due to oxide formation, there is a mass loss by erosion, confirmed by the results.

Although the mass loss by cavitation erosion in brass specimen was very close to those in the references, for a better comparison, the same conditions and materials should be used.

For the aluminum specimen, the obtained mass loss was very small, since it was expected a greater mass loss by erosion wear. Burned circular craters were also observed. Therefore, in the aluminum specimen it was not detected great mass losses, but it was possible to see the pitting formation similar to the other specimens and cavitation damage without significant mass losses is also predicted in the literature (ASM Handbook, 1992). The very small mass loss observed for these aluminum samples, was possibly due to alumina (more resistant to cavitation erosion, due to its higher hardness) formation on the surface of the specimen. Thus, the erosion mechanism in the aluminum was caused by water micro-jet impact perpendicular to the specimen surface with very low loss of mass by roughening the surface by plastic deformation, and producing the erosion crater or pit by breaking the alumina film formation in the surface.

\section{ACKNOWLEDGEMENTS}

The authors would like to gratefully thank the financial support received from FAPESC (Foundation for Research Support of the Santa Catarina State). The authors also wish to thank Mr. Roberto Schneider and the Schneider Industries for technical support, as well as the worthfull suggestions.

\section{REFERENCES}

ASM Handbook, 1992, Friction, Lubrication and Wear Technology. Vol. 18.

Bazanini, G., 2003, Temperature Calculation Inside Collapsing Bubbles in Compressible Liquids, $2^{\text {nd }}$ Brazilian Congress on Manufacturing Engineering, Uberlandia, Brazil.

Bazanini, G. and Hoays S. H., 2008, Oscilações de Cavidades na Forma Adimensionalizada. Primeiro Encontro Brasileiro sobre Ebulição, Condensação e Escoamento Multifásico LíquidoGás, Florianópolis, SC.

Fujikawa, S. and Akamatsu, T., 1980, On the Mechanisms of Cavitation Bubble Collapse, IAHR Symposium, Tokyo, Japan.
Hammitt, F. G., 1980, Cavitation and Multiphase Flow Phenomena, McGraw-Hill Book Company.

Hattori, S. and Kishimoto, M., 2008, Prediction of Cavitation Erosion on Stainless Steel Components in Centrifugal Pumps, Wear, In Press.

Janakiram, K. S., 1973, Studies on Erosion Due to Liquid Jet Impingement. MSc. Thesis, Indian Institute of Science, Bangalore.

Knapp, R. P., Daily, J. W. and Hammitt, F. G., 1970, Cavitation, McGraw-Hill.

Rao, P. V., Rao, B. C. S. and Rao, N. S. L., 1980, Erosion and Cavity Characteristics in Rotating Components, Journal of Testing and Evaluation. American Society of Testing and Materials.

Shervani-Tabar, M. T., Rezaee-Barmi, A. and Mahmoudi, S. M. S., 2003, Velocity Field and Pressure Distribution Around Two Parts of a Cavitation Bubble After its Splitting Near a Rigid Boundary, Fifth International Symposium on Cavitation, Osaka, Japan.

Soyama, H., Kumano, H. and Saka, M., 2001, A New Parameter to Predict Erosion, Fourth International Symposium on Cavitation, Pasadena, Ca., USA.

Steller, J., Krella, A, Koronowicz, J. and Janicki, W., 2005, Towards Quantitative Assessment of Material Resistance to Cavitation, Erosion, Wear, 258, pp. 604-613.

Vivekananda, P., 1983, Mechanism of Cavitation Damage Influence of Stacking Fault Energy on Erosion and Erosion Resistance of Steels and Coatings. Ph.D. Thesis, Dept. of Civil Engineering, Indian Institute of Science, Bangalore, India.

Wood, G. W., Knudsen, L. K. and Hammitt, F. G., 1967, Cavitation Damage Studies with Rotating Disk in Water. Transactions of the ASME. Journal of Basic Engineering.

Young, F. R., 1989, Cavitation, McGraw-Hill Book Company, 1989.

Zhiye, J., 1983, An Experimental Investigation on Cavitation Erosion for Propeller Alloys, China Ship Scientific Research Center Report, China. 